
allemande

46-2 | 2014

Intellectuels et politique en Allemagne

Cogestion et societas europaea - Une étude comparée en droit allemand, autrichien, belge, français, luxembourgeois et suisse

Chronique juridique

Sandie Calme

\title{
OpenEdition
}

Journals

Édition électronique

URL : https://journals.openedition.org/allemagne/1872

DOI : 10.4000/allemagne.1872

ISSN : 2605-7913

Éditeur

Société d'études allemandes

Édition imprimée

Date de publication : 30 décembre 2014

Pagination : 497-505

ISSN : 0035-0974

Référence électronique

Sandie Calme, "Cogestion et societas europaea - Une étude comparée en droit allemand, autrichien, belge, français, luxembourgeois et suisse ", Revue d'Allemagne et des pays de langue allemande [En ligne], 46-2 | 2014, mis en ligne le 29 juillet 2019, consulté le 31 mai 2021. URL : http:// journals.openedition.org/allemagne/1872 ; DOI : https://doi.org/10.4000/allemagne.1872 


\section{Chronique juridique}

\section{Cogestion et societas europaea}

Une étude comparée en droit allemand, autrichien, belge, français, luxembourgeois et suisse

\section{- Sandie Calme*}

La cogestion est à la base une spécificité normative dont le droit des sociétés allemand est une figure emblématique. La question d'implication des travailleurs au sein de l'entreprise qu'elle recouvre a été un enjeu juridique majeur dans l'élaboration de la règlementation propre à la société anonyme européenne (societas europaea, SE), avec pour perspective de préserver les droits reconnus aux salariés par les ordres juridiques nationaux intracommunautaires dans le cadre de la constitution des $\mathrm{SE}^{(1)}$. La loi de sécurisation de l'emploi du 14 juin 2013 apporte à la conceptualisation de la cogestion de manière à modifier la configuration de sociétés internationales. L'analyse comparative de ce concept nous conduit nécessairement à esquisser ce que peut être l'impact de la réforme française pour ce qui concerne nos entreprises revêtant un caractère international. Nous le constatons notamment à travers l'étude du droit allemand, autrichien, belge, français, luxembourgeois et suisse et au regard de la SE.

\section{La cogestion de droit français}

La loi de sécurisation de l'emploi étend la portée de la cogestion à des sociétés privées plus répandues que les classiques sociétés anonymes à participation ouvrière. En cela, elle offre à la cogestion un renouveau.

* Docteur en droit, LL.M. (Francfort-sur-le-Main, Allemagne), avocate au Barreau de Paris.

1 Cf. Friedrich KüBlER, « Mitbestimmungsfeindlicher Missbrauch der Societas Europaea? », in: Reinhard DАмм (dir.), Festschrift für Thomas Raiser, Berlin, de Gruyter Verlag, 2005, p. 247 sqq. 


\section{A. L'apport de la loi sur la sécurisation de l'emploi}

La loi no 2013-504 du 14 juin 2013 sur la sécurisation de l'emploi prévoit l'élection ou la désignation de représentants des salariés aux conseils d'administration et de surveillance avec le même droit de vote que les autres membres, en ce qui concerne les sociétés anonymes et les sociétés en commandite par actions d'une certaine taille: localisées en France, les sociétés concernées devront compter au moins 5000 salariés permanents, 10000 si elles sont localisées à la fois en France et à l'étranger, pourvu qu'elles soient soumises à l'obligation d'établir un comité d'entreprise d'après le Code du travail français. Dès lors qu’une société est soumise à cette obligation de représentation des salariés aux conseils d'administration et de surveillance, ses filiales en sont dispensées. Au sein d'un conseil de plus de douze administrateurs, il faudra au moins deux représentants des salariés et au moins un en présence de douze administrateurs ou moins: les statuts de la société doivent disposer du nombre d'administrateurs représentant les salariés aux conseils de surveillance ou d'administration en se conformant à ces exigences quantitatives minimales. Ces minimas sont étendus aux sociétés du secteur public et aux sociétés privatisées déjà soumises à de telles exigences de présence de représentation des salariés avec droit de vote aux conseils d'administration et de surveillance d'après l'article 5 de la loi no ${ }^{\circ}$ 83-675 sur la démocratisation du service public et d'après la loi $\mathrm{n}^{\circ} 86-912$ relative aux modalités des privatisations, ces deux lois qui ont été modifiées par l'ordonnance n 2014-948 du 20 août 2014.

\section{B. La société anonyme à participation ouvrière comme mécanisme résiduel de cogestion en droit français}

Cette forme sociale souvent méconnue qu'est la société anonyme à participation ouvrière est à même d'intéresser l'organisation des sociétés transnationales telles que la societas europaea, étant donné que pour toute société anonyme, il est possible de modifier ses statuts en assemblée générale extraordinaire afin de lui conférer la forme de société anonyme à participation ouvrière (article L225-258 du Code de commerce), cette qualité lui permettant d'émettre des actions de travail (article L225-260 $2^{\circ}$ du Code de commerce). À cet égard, il convient d'en présenter le fonctionnement.

Selon l'article L225-261 du Code de commerce, les actions de travail sont la propriété collective du personnel salarié réuni en société commerciale coopérative de main-d'œuvre comprenant obligatoirement et exclusivement les salariés liés à l'entreprise depuis au moins un an et âgés de plus de dix-huit ans. Les actions de travail ne sauraient être attribuées individuellement et en ce qui concerne la perte d'emploi, elle a pour effet l'extinction des droits de l'employé concerné dans la coopérative de main-d'œuvre.

La perte de l'emploi salarié prive le participant, sans indemnité, de tous ses droits dans la coopérative de main-d'œuvre. La liquidation des droits qui ont été acquis dans l'entreprise par l'intéressé antérieurement à son départ, au cours du dernier exercice, est faite compte tenu du temps passé par lui au cours de cet exercice, et des dispositions de l'article L225-269 (article L225-261 alinéa 1 du Code de commerce).

En aucun cas les actions de travail ne peuvent être attribuées individuellement aux salariés de la société, membres de la coopérative de main-d'œuvre (article L225-261 alinéa 4 du Code de commerce). 
Les actions de travail, nominatives et inscrites au nom de la société coopérative de main-d'œuvre, sont inaliénables pendant toute la durée de la société à participation ouvrière (article L225-262 du Code de commerce).

Les membres de la société coopérative de main-d'œuvre élisent parmi eux leurs représentants au sein des assemblées générales, le nombre de voix de ces mandataires relevant de la proportion des actions de travail conformément aux statuts (article L225-263 du Code de commerce).

Les participants à la société coopérative de main-d'œuvre sont représentés aux assemblées générales de la société anonyme par des mandataires élus par ces participants, réunis en assemblée générale de la coopérative (article L225-263 alinéa $1^{\text {er }}$ du Code de commerce).

Les mandataires élus doivent être choisis parmi les participants. Leur nombre est fixé par les statuts de la société anonyme (article L225-263 alinéa 2 du Code de commerce).

Le nombre des voix dont disposent ces mandataires, à chaque assemblée générale de la société anonyme, est établi d'après le nombre de voix dont disposent les autres actionnaires présents ou représentés, en respectant la proportion entre les actions de travail et les actions de capital résultant de l'application des statuts de la société. Il est déterminé au début de chaque assemblée d'après les indications de la feuille de présence (article L225-263 alinéa 3 du Code de commerce).

Le conseil d'administration de la société anonyme à participation ouvrière comporte un ou plusieurs représentants de la société coopérative de main-d'œuvre, élus par l'assemblée générale des actionnaires et choisis parmi les mandataires qui représentent la coopérative à cette assemblée générale, dans une proportion correspondant à la quotité d'actions du travail. Nommés pour le même temps que les autres administrateurs, ces représentants sont rééligibles, même si leur mandat prend fin s'ils cessent d'être salariés et membres de la société (article L225-268 du Code de commerce).

Si le conseil d'administration ne se compose que de trois membres, il doit comprendre tout au moins un représentant de ladite société coopérative (article L225-268 alinéa $1^{\text {er }}$ du Code de commerce).

Toutefois, une gradation est opérée entre les employés quant à leur ancienneté et en ce qui concerne les motifs de leur départ, pour ce qui est de la répartition des parts sociales entre les salariés en cas de dissolution de la société. En effet, l'article L225269 du Code de commerce précise que la répartition se fait seulement « entre les participants et anciens participants comptant au moins dix ans de services consécutifs dans les établissements de la société, ou tout au moins une durée de services sans interruption égale à la moitié de la durée de la société, et ayant quitté la société pour l'une des raisons suivantes: départ à la retraite volontaire ou d'office avec droit à pension, maladie ou invalidité entraînant l'inaptitude à l'emploi précédemment occupé, licenciement motivé par une suppression d'emploi ou une compression de personnel ». Ainsi organisé, ce système semble avoir pour vocation première d'intéresser et de récompenser la fidélité des salariés à la société en sanctionnant les salariés les plus récemment engagés ainsi que ceux dont le départ de la société n'est pas détaché de toute idée de faute professionnelle (article L225-268 alinéa 2 du Code de commerce). 
En cas de dissolution, l'actif social n'est réparti entre les actionnaires qu'après l'amortissement intégral des actions de capital (article L225-269 alinéa $1^{\text {er }}$ du Code de commerce).

Les administrateurs côté travailleurs, qui doivent effectivement être en emploi auprès de la société, ne sauraient représenter plus du tiers des administrateurs en fonction, sans compter les représentants de la coopérative de main-d'œuvre (article L225$22 \mathrm{du}$ Code de commerce). Cet article précise (article L225-22 alinéas 5 et 6 du Code de commerce) - et cela peut présenter un intérêt pour le cas de la société anonyme européenne - qu'en cas de fusion (ou de scission), le contrat de travail peut avoir été conclu avec l'une des sociétés fusionnées (ou avec la société scindée).

Observons, pour ce qui est de la comparaison avec le système allemand, que la société anonyme ouvrière relève, selon la lettre du texte, du système moniste. Il en résulte que le système de représentation des salariés au sein du conseil de surveillance, propre à l'ordre juridique allemand, est bien distinct.

Peu représentatif du fonctionnement actuel des sociétés anonymes françaises, le système des sociétés anonymes à participation ouvrière va bien au-delà des modalités classiques de représentation des intérêts des travailleurs au sein de l'entreprise.

Ces mécanismes de cogestion spécifiques au droit français peuvent présenter des affinités avec la cogestion de droit allemand, modèle repris assez fidèlement en droit autrichien et en droit luxembourgeois.

\section{La cogestion de droit allemand, de droit autrichien et de droit luxembourgeois, un modèle qui se présente comme précédant la réforme de droit français}

Le droit allemand des sociétés, pionnier de la cogestion au sens strict, se caractérise par la prégnance du système dualiste, avec directoire et conseil de surveillance, quant à la structure des sociétés. Ce système dualiste a également connu un certain succès en droit français, qui permet aujourd'hui de choisir entre les deux types de structures moniste (société anonyme avec président directeur général et conseil d'administration) et dualiste. Si le droit allemand connaît un système de représentation des entreprises similaire à celui du comité d'entreprise que l'on retrouve dans nombre de sociétés de l'Union européenne, et qui constitue une forme secondaire de cogestion, la spécificité juridique allemande réside dans la cogestion à proprement parler $^{(2)}$, qui permet une véritable prise de décision des travailleurs dans l'entreprise.

La cogestion (Mitbestimmung) au sens strict ${ }^{(3)}$ est une modalité structurale qui concerne les sociétés de capitaux en fonction de leur effectif, de sorte que ce système de cogestion ne viendra à s'appliquer que pour des sociétés d'une certaine taille. L’enjeu est important puisqu'une implication plus ou moins étroite des salariés dans la gestion de l'entreprise est en cause. Le lieu d'exercice de la cogestion est le conseil de surveillance, typique du système dualiste. En son sein, on retrouve tant des représentants

2 Cf. Dr. Günter Halbach, Alfred Mertens, Dr. Rolf Schwedes, Prof. Dr. Otfried Wlotzke, Übersicht über das Recht der Arbeit, Verlag Bundesministerium für Arbeit und Sozialordnung Bonn, 1987 (2 éd.).

3 Dr. Rainer Knieast, in: Dr. Dirk Jannott et Dr. Jürgen Frodermann (dir.), Handbuch der Europäischen Aktiengesellschaft - Societas Europaea, C.F. Müller Campus, 2005, ch. 13, p. 384 sqq. 
des détenteurs de parts sociales que des représentants des salariés, pourvu que la société en cause occupe plus de 500 salariés. En ce qui concerne les sociétés de plus de 500 salariés, c'est la loi Drittelbeteiligungsgesetz de 2004 qui trouve à s'appliquer, conduisant ainsi à l'élection d'un tiers des membres du conseil de surveillance par les salariés. Un degré de cogestion plus élevé est atteint pour plus de 1000 salariés dans le domaine métallurgique avec la loi Mitbestimmungsgesetz de 1951. Dans ce cadre, la parité au sein du conseil de surveillance est atteinte puisque, paritairement composé de représentants des détenteurs de parts sociales et des travailleurs, il est organisé de telle sorte qu'en cas de partage des voix du fait de cette parité, un membre neutre choisi par les deux parties devra être nommé qui tranchera la question en suspens. Le domaine métallurgique semble ainsi particulièrement privilégié. D’autant plus que les sociétés de capitaux ordinaires (en dehors du domaine métallurgique) de plus de 2000 salariés disposeront d'un conseil de surveillance paritaire mais avec, en cas de partage des voix, un droit de veto exercé par le président du conseil de surveillance obligé envers les détenteurs du capital de l'entreprise.

En droit autrichien, il est prévu que dans les sociétés anonymes et entreprises assimilées, le comité central d'entreprise ou le comité d'entreprise désigne un tiers des membres du conseil de surveillance pour représenter les travailleurs (article $\$ 110$ de la loi sur l'organisation du travail, Arbeitsverfassungsgesetz).

Le système à l'origine allemand de la cogestion a été repris en droit luxembourgeois du travail pour les sociétés anonymes, et ce selon un système unique et élaboré intégré au sein du Code du travail luxembourgeois en 2006, en ses articles L426-1 et suivants, prévoyant désormais qu'un tiers des administrateurs ou membres du conseil de surveillance de sociétés anonymes d'une certaine taille doit représenter le personnel de l'entreprise (article L426-3 du Code du travail luxembourgeois).

\section{La cogestion au sens strict est inconnue en droit belge et en droit suisse}

En droit belge, on ne retrouve pas de véritable système de cogestion ${ }^{(4)}$, l'implication des salariés dans les unités techniques d'exploitation ou entreprises d'une certaine taille étant réduite à l'établissement d'un conseil d'entreprise dont les attributions se limitent surtout aux questions sociales ${ }^{(5)}$; le conseil d'entreprise est informé et consulté sur tous les projets généraux susceptibles d'avoir des conséquences sur l'organisation ou sur les conditions de travail, il établit le règlement intérieur, administre les affaires sociales, détermine les plans de congés et il est tenu informé de la situation économique de l'entreprise grâce aux renseignements généraux qu'il reçoit régulièrement, ses attributions relevant précisément de l'article 15 de la loi du 20 septembre 1948 portant organisation de l'économie, loi qui, en sa section IV, régit les conseils d'entreprise.

La loi fédérale suisse sur l'information et la consultation des travailleurs dans les entreprises, dite loi sur la participation, entrée en vigueur le $1^{\mathrm{er}}$ mai 1994, régit la question de l'implication des travailleurs au sein de toutes les entreprises privées qui, en

4 Cf. Jacques-Louis Colombani et Marc Favero, Societas europaea: la société européenne, SE, éd. Joly, $2002, \mathrm{n}^{\circ} 17$, p. 9.

5 Voir La participation des salariés à la gestion des entreprises, rapport au Garde des Sceaux, juin 1999, Les documents de travail du Sénat, Série LÉGISLATION COMPARÉE, p. 13. 
Suisse, occupent des travailleurs en permanence (article $1^{\text {er }}$ de la loi sur la participation.). Selon l'article 9 de cette loi relatif au droit à l'information, la représentation des travailleurs a le droit d'être informée en temps opportun et de manière complète sur toutes les affaires dont la connaissance lui est nécessaire pour s'acquitter convenablement de ses tâches (article 9 alinéa $1^{\text {er }}$ de la loi sur la participation) et l'employeur est tenu d'informer la représentation des travailleurs au moins une fois par an sur les conséquences de la marche des affaires sur l'emploi et pour le personnel (article 9 alinéa 2 de la loi sur la participation).

Le droit d'être représenté par des représentants élus par les travailleurs et regroupés en une ou plusieurs représentations qui défendent, envers l'employeur, les intérêts communs des travailleurs et les tiennent régulièrement informés sur son activité (article 8 de la loi sur la participation) est limité aux entreprises occupant au moins cinquante travailleurs (article 3 de la loi sur la participation). Dans les entreprises ou secteurs d'entreprise sans représentation des travailleurs, ce sont ces mêmes travailleurs qui exercent directement le droit à l'information tout comme le droit à la participation (article 4 de la loi sur la participation).

L'article 10 de la loi cantonne les droits de participation aux domaines de la sécurité au travail, du transfert de l'entreprise, des licenciements collectifs, de l'affiliation à une institution de la prévoyance professionnelle et de la résiliation d'un contrat d'affiliation. Selon l'appréciation doctrinale, cette loi sur la participation ne prescrit aucune réelle implication des travailleurs dans les organes, dans la mesure où elle se cantonne à la communication d'informations et à une participation dans des domaines portant sur le champ d'activité des travailleurs ${ }^{(6)}$.

\section{L'implication internationale de la cogestion : l'exemple de la société anonyme européenne}

La mise en perspective de différents systèmes nationaux de cogestion révèle l'implication internationale que peut receler la règlementation d'un tel concept. Les ordres juridiques internes ont leur propre conception de la cogestion au sein de la SE, cogestion qui s'articule avec leur propre appréhension de la cogestion pour les sociétés anonymes nationales. Le droit suisse pourrait s'offrir, par l'impulsion de la doctrine, son propre système de cogestion pour un SE de droit suisse à venir.

\section{A. Le système de cogestion de la SE: un système basé sur les dispositions tant générales que spéciales des droits nationaux}

À la base, la Société anonyme européenne sera immatriculée dans un seul État membre où elle aura son siège statutaire et son administration centrale (article $7 \mathrm{du}$ règlement SE). Toutefois, elle ne sera pas soumise à un régime juridique purement supranational, puisqu'elle relèvera en partie du régime juridique de son État d'immatriculation. Observons que toute SE est affectée d'un système d'implication des travailleurs prédéfini et réglementé ${ }^{(7)}$.

6 Maiko Günther, La société anonyme européenne. Prévention, critique et perspectives de mise en œuvre par la Suisse, Bâle, Helbing Lichtenhahn, 2009, p. 293.

7 Cf. Colombani/Favero, Societas europaea (note 4), nº 70, p. 23. 
La doctrine considère la question de la diversité( ${ }^{(8)}$ comme un problème majeur du droit européen des sociétés et qualifie la volonté de protéger les salariés comme légitime ${ }^{(9)}$. Toutefois, il semble que la réglementation européenne du droit des sociétés aille dans le sens de la flexibilité en s'appuyant sur des conceptions du modèle anglo-saxon, dans la mesure, par exemple, de la communication de la Commission européenne du 21 mai 2003 intitulée «Modernisation du droit des sociétés et renforcement du gouvernement d'entreprise dans l'Union européenne. Un plan pour avancer " ${ }^{(10)}$.

La possibilité pour chaque État membre de prendre des dispositions juridiques propres à la SE a pu être considérée comme une tentation de "dumping juridique » où les États membres introduiraient dans leur ordre juridique des normes dotant les SE nationales d'un régime juridique attractif dans un esprit de compétition entre les droits nationaux ${ }^{(11)}$.

La pratique ayant besoin de flexibilité, en particulier quant aux montages d'ingénierie juridique propres à la $\mathrm{SE}$, et le règlement n'ayant pas fait, selon la doctrine, la part belle à la liberté contractuelle, le degré de flexibilité des règles nationales applicables à la SE a été présenté comme un facteur du choix de la localisation géographique des futures sociétés européennes ${ }^{(12)}$.

Les règles relatives à l'implication des travailleurs dans la SE font l'objet de la directive 2001/86/CE du Conseil du 8 octobre 2001 complétant le statut de la société européenne pour ce qui concerne l'implication des travailleurs, cette directive et le règlement formant un tout indissociable. Les questions laissées en suspens par la directive sont réglées par le droit national des États membres relatif aux sociétés anonymes. Pour ce qui concerne l'information, la consultation et la participation des travailleurs au niveau transnational, la conciliation passe au premier plan $^{(13)}$ des règles subsidiaires intervenant à défaut d'accord entre les personnes concernées. Selon le concept avant-après, il importe beaucoup que les droits des travailleurs existant avant la constitution des SE soient à la base de l'aménagement de leurs droits en matière d'implication dans la SE.

Si le système de la conciliation joue un rôle primordial facteur de flexibilité, les droits nationaux jouent un rôle essentiel, aussi bien concernant les normes nationales applicables spécialement à la configuration de la société anonyme européenne que pour ce qui est des dispositions applicables aux sociétés anonymes locales.

En droit allemand, l'implication des travailleurs au sein de la Société anonyme européenne relève de la loi Gesetz über die Beteiligung der Arbeitnehmer in einer Europäischen Gesellschaft (SE-Beteiligungsgesetz-SEBG).

En droit autrichien, la question de l'implication des travailleurs dans la Société anonyme européenne est régie par la loi Gesetz über das Statut der Europäischen Gesellschaft (SE-Gesetz - SEG), qui est entrée en vigueur le 8 octobre 2004.

8 Cf. ibid., $\mathrm{n}^{\circ} 19$, p. 9 sqq.

9 Jean-Pierre Bertrel, La société européenne, Societas europaea, SE, éd. par l'École supérieure de management, Chambre de commerce et d'industrie, 2004, p. 11.

10 Ibid., p. 12.

11 Ibid., p. 13.

12 Ibid., p. 14.

13 Cf. Colombani/Favero, Societas europaea (note 4), n 150, p. 46. 
Le droit belge a choisi la voie de la réglementation par convention collective, avec la convention collective de travail $n^{\circ} 84$ du 6 octobre 2004, conclue au sein du Conseil national du Travail, concernant l'implication des travailleurs dans la Société européenne, rendue obligatoire par arrêté royal entré en vigueur le 19 janvier 2005. Cette convention collective a été modifiée par la convention collective $\mathrm{n}^{\circ} 84$ bis du 21 décembre 2010, entrée en vigueur le 8 avril 2011.

Le droit français réglemente la question de l'implication des travailleurs au sein de la SE aux articles L2351-1 et suivants du Code du travail.

En droit luxembourgeois, c'est la loi du 25 août 2006 qui a complété le statut de la SE pour ce qui concerne l'implication des travailleurs. Ses dispositions ont été insérées aux articles L441-1 et suivants du Code du travail par le règlement grand-ducal du 22 décembre 2006 procédant à la consolidation du Code du travail.

Dans ce cadre, on trouve une véritable articulation entre normes nationales propres à la $\mathrm{SE}$ et normes nationales plus générales. Ainsi, un système de cogestion élaboré tel que ceux du droit allemand, du droit autrichien, du droit luxembourgeois voire du droit français sur la société anonyme à participation ouvrière, systèmes très précisément réglementés pouvant laisser craindre une inflation législative, vient à s'imposer dans le détail pour ce qui est de la règle avant-après. À l'inverse, il n'y a pas de système national de cogestion à préserver en droit belge comme en droit suisse. Cela peut expliquer, dans ce cadre, l'instauration d'une certaine flexibilité.

\section{B. Le système de cogestion de la SE: vers une adaptation possible en droit suisse?}

Le droit suisse ne relève pas, a priori, des dispositions communautaires relatives à la Société anonyme européenne. Néanmoins, une SE peut être liée à la Suisse par des formes de coopération telles que l'implantation des filiales, de succursales, la fusion, par le transfert du siège social effectif ou le mécanisme de reconnaissance de la société étrangère en Suisse ${ }^{(14)}$.

La doctrine lance une proposition ${ }^{(15)}$ de loi fédérale supposant une adhésion par la Suisse au règlement $\mathrm{CE} \mathrm{n}^{\circ}$ 2157/2001 relatif au statut de la Société Européenne (SE). Maiko Günther propose une loi fédérale complétant le règlement CE n 2157/2001 relatif au statut de la Société Européenne (SE) selon laquelle «Conformément 4 du règlement-SE, selon lequel les statuts de la SE ne doivent à aucun moment entrer en conflit avec les modalités relatives à l'implication des travailleurs, notamment en cas de nouvelles négociations dans le cadre de la directive-SE, le conseil d'administration ou l'organe de direction peut apporter les modifications nécessaires aux statuts sans nouvelle décision de l'assemblée générale » (article 6). L’usage de cette faculté offerte par le règlement SE en son article 12,4 , alinéa $1^{\mathrm{er}}$, pour un État membre, de prévoir que l'organe de direction ou l'organe d'administration de la SE ait le droit d'apporter des modifications aux statuts sans nouvelle décision de l'assemblée générale des actionnaires, afin que les statuts de la SE n'entrent pas en conflit avec les modalités relatives à l'implication des travailleurs, une modification des statuts étant nécessaire lorsque de nouvelles modalités sont fixées selon la directive 2001/56/CE, se retrouve déjà en droit

14 M. GüNTHER, La société anonyme européenne (note 6), p. 272.

15 Ibid., p. 298. 
positif belge, selon lequel, dans le cas prévu à l'article 12,4 , alinéa $1^{\text {er }}$, du règlement (CE) $n^{\circ} 2157 / 2001$, le conseil d'administration ou le conseil de direction a le droit d'apporter des modifications aux statuts sans nouvelle décision de l'assemblée générale des actionnaires. Cette similitude entre le droit belge et la proposition doctrinale pour le droit suisse peut être analysée en parallèle avec la spécificité d'absence de toute forme élaborée de cogestion tant en droit suisse qu'en droit belge. 